\title{
Der Einfluss des Bildungsniveaus von Frauen auf das Scheidungsverhalten in der Schweiz, 1952-2013
}

\author{
Simone Villiger*
}

Zusammenfassung: Der Beitrag zeigt auf, dass von 1952 bis 2003 in der Schweiz für hoch gebildete Frauen die höchste Scheidungswahrscheinlichkeit bestand. Von 2004 bis 2013 hingegen sind keine bildungsspezifischen Unterschiede hinsichtlich des Scheidungsverhaltens mehr festzustellen, da die Scheidungswahrscheinlichkeit vor allem für tief, aber auch für mittel gebildete Frauen zugenommen hat. Folglich ist der Zusammenhang zwischen dem Bildungsniveau der Frauen und dem Scheidungsverhalten in der Schweiz zwischen 1952 und 2013 verschwunden.

Schlüsselwörter: Scheidung, Bildung, soziale Ungleichheit, Ereignisanalyse, Schweiz

\section{L'influence de la formation des femmes sur le divorce en Suisse, 1952-2013}

Résumé: Cet article montre qu'entre 1952 et 2003, les femmes ayant un haut niveau de formation présentaient la plus forte probabilité de divorce. En revanche, l'effet du niveau de formation des femmes sur la divortialité n'est plus observé entre 2004 et 2013, étant donné que la probabilité de divorce a augmenté notamment chez les femmes ayant un bas niveau de formation, mais aussi chez celles ayant un niveau de formation moyen. Ainsi, le lien entre le niveau de formation des femmes et la divortialité en Suisse a disparu entre 1952 et 2013. Mots-clés: divorce, formation, inégalités sociales, analyse des événements, Suisse

\section{The Impact of Female Education on Divorce in Switzerland, 1952-2013}

Abstract: This article shows that from 1952 to 2003 the risk of divorce in Switzerland was highest for highly educated women. From 2004 to 2013, however, no association between female education and risk of divorce can be found, primarily because the divorce risk of low educated women and - less pronounced - for women with middle grade education have increased. Therefore, between 1952 and 2013, the association between female education and risk of divorce in Switzerland has disappeared.

Keywords: divorce, education, social inequality, time-event-analysis, Switzerland

\section{$1 \quad$ Einleitung}

Zu den zentralen Aspekten des familialen Wandels in Europa im 20. Jahrhundert gehört sicherlich der starke Anstieg der Scheidungsrate. Die Zunahme war in den nordischen Ländern am höchsten, gefolgt von den westlichen sowie den mittel- und osteuropäischen Staaten (Sobotka und Toulemon 2008). In den mediterranen Län-

Soziologisches Institut, Universität Zürich, CH-8050 Zürich, simone.villiger@gmail.com. 
dern ist die Scheidungsrate heute am tiefsten, aber auch dort ist sie in den letzten Jahrzehnten deutlich angestiegen (Bernardi und Martinez-Pastor 2010).

Eine Vielzahl an Studien ist den Gründen für diese Entwicklung nachgegangen und hat versucht, die Faktoren zu benennen, die das Scheidungsverhalten beeinflussen. Dem Bildungsniveau der Frauen, welches in allen industrialisierten Ländern in den letzten Jahrzehnten gestiegen ist (Blossfeld und Shavit 1993), wurde hierbei besondere Aufmerksamkeit geschenkt (Blossfeld et al. 1995). Es gilt als Indikator für den jeweils individuellen sozialen Status, das Einkommenspotenzial, die intellektuellen Fähigkeiten, aber auch als Gradmesser für individuelle Autonomie und die Unabhängigkeit von sozialen Normen (Matysiak et al. 2014).

In der Literatur wird postuliert, dass sich bildungsspezifische Unterschiede im Scheidungsverhalten niederschlagen und dass diese Korrelation in den letzten Jahrzehnten bei den Frauen einem Wandlungsprozess unterworfen war. Die unter anderem auf Goodes Theorie der sich verändernden sozialen Determinanten einer Scheidung (Goode 1962; Goode 1970) sowie auf neueren theoretischen Ansätzen zur Transformation des Ehemodells von der spezialisierten zur symmetrischen Rollenverteilung (Isen und Stevenson 2010; Chen 2012) basierende These lautet, dass der Nutzen einer Ehe für höher gebildete Frauen lange Zeit kleiner war als für niedriger Gebildete und die Scheidungskosten zudem so hoch waren, dass sich nur diejenigen einen solchen Schritt leisten konnten, die über die entsprechenden Ressourcen verfügten, also primär höher gebildete Frauen. Als Konsequenz war die Scheidungswahrscheinlichkeit für höher gebildete Frauen lange Zeit am höchsten. Aufgrund rechtlicher, institutioneller, ökonomischer, kultureller und sozialer Kontextfaktoren, die sich im Verlauf der letzten Jahrzehnte verändert haben - Liberalisierung des Scheidungsrechts, steigende Arbeitsmarktbeteiligung der Frauen, Ausbau der wohlfahrtsstaatlichen Leistungen, Abnahme der Religiosität, zunehmende Verbreitung und Akzeptanz neuer Lebensformen, eine gesellschaftliche Tendenz in Richtung egalitäres Genderregime sowie die Entwicklung eines Ehemodells weg von der Spezialisierung hin zur Symmetrie -, ist der Nutzen einer Ehe für höher Gebildete gestiegen, während die Scheidungskosten gesunken sind (Goode 1962; Goode 1970; Becker 1981; Blossfeld et al. 1995; Isen und Stevenson 2010; Chen 2012). In der Folge haben sich immer weniger gut gebildete Frauen und immer mehr niedriger gebildete Frauen scheiden lassen, sodass sich der konstatierte Zusammenhang zwischen dem Bildungsniveau der Frauen und dem Scheidungsverhalten abgeschwächt hat, dann verschwunden ist und sich letztlich ins Gegenteil verkehrt hat, was heisst, dass sich heutzutage niedriger gebildete Frauen am häufigsten scheiden lassen (Goode 1962; Blossfeld et al. 1995; Härkönen und Dronkers 2006). ${ }^{1}$

Für Europa sind nur wenige Studien greifbar, die explizit untersucht haben, ob sich der Zusammenhang zwischen dem Bildungsniveau der Frauen und dem

1 In der Literatur wird nicht spezifiziert, wann genau der Zusammenhang verschwindet bzw. wann er sich ins Gegenteil verkehrt, was denn auch kritisiert wurde (De Graaf und Kalmijn 2006). 
Scheidungsverhalten in den letzten Jahrzehnten ins Gegenteil verkehrt hat (De Graaf und Kalmijn 2006). Die Resultate sind gemischt: Es gibt Länder wie Grossbritannien und Schweden, in denen sich eine Umkehrung des Zusammenhangs zeigt (Hoem 1997; Chan und Halpin 2002); in Ländern wie Italien und Frankreich hat sich der Zusammenhang abgeschwächt (Härkönen und Dronkers 2006; Salvini und Vignoni 2011); Länder wie Österreich, Deutschland und die Schweiz weisen keine Veränderung auf (Härkönen und Dronkers 2006) und für Länder wie die Niederlande, Spanien und Finnland liegen widersprüchliche Resultate vor (Portman und Kalmijn 2002; Härkönen 2005; De Graaf und Kalmijn 2006; Härkönen und Dronkers 2006; Bernardi und Martinez-Pastor 2010).

Eine Erklärung für diese gemischten Resultate ist, dass sich die Kontextfaktoren zwar in den meisten europäischen Ländern dahingehend verändern, dass die Scheidungskosten sinken, der Nutzen einer Ehe für besser gebildete Frauen steigt und sich der Zusammenhang zwischen dem Bildungsniveau der Frau und dem Scheidungsverhalten folglich wie oben beschrieben verändert, dass dieser Prozess aber je nach Länderkontext unterschiedlich schnell vonstatten geht (Blossfeld et al. 1995). ${ }^{2}$

Die meisten der oben genannten Studien basieren auf Daten aus den 1990erJahren. Die Kontextfaktoren, die einen Einfluss auf die Scheidungskosten bzw. auf den Nutzen einer Ehe haben und dadurch die bildungsspezifischen Ungleichheiten im Scheidungsverhalten von Frauen beeinflussen, sind aber nach wie vor im Wandel begriffen. Um zu prüfen, ob die oben beschriebene Veränderung der bildungsspezifischen Unterschiede im Scheidungsverhalten als universeller Prozess zu werten ist, der sich in allen europäischen Ländern früher oder später vollzieht, bietet es sich an, diejenigen Länder, in denen sich bis in die 1990er-Jahre noch keine Umkehrung des Zusammenhangs gezeigt hat, erneut auf der Grundlage aktueller Daten zu untersuchen.

Der vorliegende Artikel untersucht mit Daten aus dem Jahr 2013 die bildungsspezifischen Unterschiede im Scheidungsverhalten in der Schweiz - ein Land, das diesbezüglich im Vergleich zu anderen europäischen Staaten noch relativ unerforscht ist. Es soll der Frage nachgegangen werden, ob sich die bildungsspezifischen Unterschiede im Scheidungsverhalten für Frauen zwischen 1952 und 2013 verändert haben.

In der Schweiz waren in den letzten Jahrzehnten vor allem jene Kontextfaktoren einem Wandlungsprozess unterworfen, die einen Einfluss auf die Scheidungskosten haben: Das Scheidungsrecht wurde im Jahr 2000 liberalisiert (BFS 2009), die Religiosität hat in breiten Teilen der Bevölkerung abgenommen und die Kirche damit an Einfluss verloren (Nicolet und Tresch 2009; BFS 2015a), gleichzeitig erfolgte ein Anstieg der Erwerbsbeteiligung der Frauen und folglich deren finanziellen Unabhängigkeit (BFS 2005), und neue, heute akzeptierte Lebensformen haben sich verbreitet (Fux 2005), sodass davon ausgegangen werden kann, dass die Scheidungskosten über

2 Weitere Erklärungen sind bei den Unterschieden in der Grösse der Stichprobe, in den berücksichtigten Erklärungsvariablen und den Modellspezifikationen zu suchen. 
die Zeit hinweg gesunken sind. Wenig Veränderung zeigt sich hingegen bei jenen Kontextfaktoren, die den Nutzen einer Ehe für gut ausgebildete Frauen erhöhen: Es dominiert in der Schweiz nach wie vor ein konservatives Genderregime und die Familienpolitik ist immer noch stark auf das Ein-Ernährer-Modell ausgerichtet (Nordenmark 2013). Zwar ist aufgrund der zunehmenden Erwerbstätigkeit der Frauen das Doppelverdiener-Modell seit den 2000er-Jahren stärker verbreitet als das Ein-Ernährer-Modell (BFS 2015b), jedoch arbeiten die Frauen in der Schweiz im Vergleich mit Frauen aus anderen europäischen Ländern überdurchschnittlich häufig Teilzeit und übernehmen nach wie vor den grössten Anteil an Hausarbeit und Kinderbetreuung (Pfau-Effinger 2005). Zudem sind die egalitären Familienmodelle (beide Elternpaare arbeiten Teil- bzw. Vollzeit) weiterhin stark untervertreten (BFS 2015b). Somit kann angenommen werden, dass der Nutzen der Ehe für besser gebildete Frauen in der Schweiz in den letzten Jahrzehnten nur geringfügig angestiegen ist.

Mit Blick auf die gesunkenen Scheidungskosten und den nur geringfügig gestiegenen Nutzen einer Ehe für höher gebildete Frauen lautet die zu testende Hypothese, dass zwischen 1952 und 2013 in der Schweiz die Scheidungswahrscheinlichkeit für Frauen mit niedrigerer Bildung zu-, jene für Frauen mit hoher Bildung jedoch nicht abgenommen hat, sodass sich der Zusammenhang zwischen dem Bildungsniveau der Frauen und dem Scheidungsverhalten in der Schweiz im genannten Zeitraum abgeschwächt hat, sich aber (noch) nicht ins Gegenteil verkehrt hat.

Im Folgenden werden zunächst theoretische Argumente aufgeführt, die erläutern, welche Kontextfaktoren eine Senkung der Scheidungskosten bzw. einen Anstieg des Nutzens einer Ehe für besser gebildete Frauen bewirken (2.). Danach wird der historische Kontext der Schweiz von 1950 bis 2010 genauer beleuchtet (3.). In den anschliessenden Kapiteln gilt es, den Datensatz, die Variablen sowie das methodische Vorgehen zu beschreiben (4.), die Resultate zu erläutern (5.) und diese anschliessend zu diskutieren (6.).

\section{Theoretische Betrachtungen}

Dieser Beitrag geht in Anlehnung an Blossfeld und Mitautoren (1995) sowie an Chen (2012) davon aus, dass 1) die bildungsspezifischen Unterschiede im Scheidungsverhalten in einem Land durch ein komplexes Wechselspiel von rechtlichen, institutionellen, kulturellen, ökonomischen und sozialen Kontextfaktoren, welche einen Einfluss auf die Scheidungskosten bzw. den Nutzen einer Ehe für besser gebildete Frauen haben, beeinflusst werden und dass 2) Veränderungen in diesen Kontextfaktoren letztlich zu Veränderungen in den bildungsspezifischen Unterschieden im Scheidungsverhalten führen. ${ }^{3}$ In Übereinstimmung mit den meisten

3 Eine weitere Erklärung für die Veränderung des untersuchten Zusammenhangs ist, dass sie ein Artefakt ist, der durch die stärkere Selbstselektion gut gebildeter Frauen (z. B. aufgrund unbeob- 
Studien zum sich verändernden Scheidungsverhalten der Frauen (u.a. Cherlin 1992; Lyngstad und Jalovaara 2010; Härkönen 2013) wird ferner angenommen, dass das veränderte Scheidungsverhalten vor allem auf Perioden- und nicht auf Kohorteneffekte zurückzuführen ist, was bedeutet, dass die oben erwähnten Kontextfaktoren - zumindest potenziell - alle Ehen beeinflussen, unabhängig davon, wann sie geschlossen wurden. ${ }^{4}$

\subsection{Sinkende Scheidungskosten}

In Zeiten, in denen eine Scheidung kompliziert und in einer Gesellschaft ein seltenes Ereignis war, konnten sich nur die Mitglieder der Oberschicht und somit die Gebildeten eine Scheidung leisten. Sie verstanden das Scheidungsprozedere besser, weil sie unter anderem über ein grösseres Netzwerk für Hilfestellungen verfügten, und konnten die damit einhergehenden Kosten besser tragen. Aufgrund einer Lockerung der rechtlichen Hürden wurden die Scheidungsprozesse jedoch zunehmend zugänglich und bezahlbar für alle Schichten, sodass vor allem die weniger Gebildeten, die tendenziell mit grösseren sozialen und ökonomischen Problemen belastet und deren Ehen somit grundsätzlich instabiler sind, für höhere Scheidungsraten sorgten. Und weil sich immer mehr niedriger Gebildete scheiden liessen, so Goode (1993), hat sich der Zusammenhang zwischen dem Bildungsniveau der Frauen und dem Scheidungsverhalten ins Gegenteil verkehrt: Niedriger Gebildete lassen sich heutzutage häufiger scheiden als besser Gebildete.

Aber nicht nur die rechtlichen, auch die normativen Hürden sind gesunken. In Ländern, in denen die katholische Kirche dominant und das Familienmodell traditionell ist, stellt die Scheidung einen stärkeren Verstoss gegen die soziale Norm dar und bedeutet somit für die Betroffenen eine Stigmatisierung (Blossfeld et al. 1995). In diesen Ländern sind die normativen Scheidungskosten höher, und es braucht zusätzliche Ressourcen - wie zum Beispiel Bildung -, um sich vom sozialen Druck zu befreien. Mit den höheren Scheidungsraten in allen Bevölkerungsschichten, dem geringeren Einfluss der katholischen Kirche und dem zunehmend modernen Familiensystem nimmt gemäss Blossfeld und Mitautoren (1995) die Bedeutung des Stigmas einer Scheidung ab, die Toleranz in der Bevölkerung steigt, der «befreiende» Effekt von Bildung verliert an Bedeutung und der Zusammenhang zwischen dem Bildungsniveau und dem Scheidungsverhalten wird schwächer oder verschwindet gar. Zudem hat sich die ökonomische Situation der niedriger gebildeten Frauen verbessert. In Ländern, in denen vermehrt Frauen aus allen Schichten erwerbstätig sind und die wohlfahrtsstaatlichen Leistungen und Dienstleistungen umfangreich

achteter Charakteristika wie grosse Hingabe an die Institution Ehe), welche sich entscheiden zu heiraten, zustande kommt. Bernardi und Martinez-Pastor (2011) konnten für Spanien jedoch zeigen, dass die Selbstselektion das Resultat nicht verzerrt.

4 Kohorteneffekte (hier: Heiratskohorten) entstehen dann, wenn die Bedingungen, die bei der Eheschliessung vorherrschten, die Erwartungen und das Verhalten während der ganzen Ehe beeinflussen (Härkönen 2013). 
und grosszügig sind, können zunehmend alle Frauen, auch die niedriger Gebildeten, sich und ihre Kinder unabhängig von ihrem Ehemann durchbringen. Oppenheimer (1997) hingegen führt an, dass umfangreiche und grosszügige wohlfahrtsstaatliche Leistungen und Dienstleistungen die ökonomische Belastung der Paare und Familien reduziere und daher stabilisierend insbesondere auf Ehen wirke, welche tendenziell mehr finanzielle Schwierigkeiten haben, also jene von niedriger Gebildeten.

Um die Entwicklung der bildungsspezifischen Unterschiede im Scheidungsverhalten über die Zeit hinweg besser zu verstehen, muss der Blick aber nicht nur auf die gesunkenen Scheidungskosten und auf den damit einhergehenden Anstieg der Scheidungsrate bei den niedriger gebildeten Frauen gerichtet werden, sondern auch auf einen sich verändernden gesellschaftlichen Kontext in Richtung Genderegalitarismus, sowohl in der Gesellschaft wie auch innerhalb der Ehe (Chen 2012). Diese Veränderung hat, wie im nächsten Kapitel ausgeführt wird, zur Konsequenz, dass der Nutzen einer Ehe im Vergleich mit dem Singleleben für besser gebildete Frauen ansteigt, sodass deren Ehen an Stabilität gewinnen.

\subsection{Gestiegener Nutzen der Ehe für besser gebildete Frauen}

Lange Zeit galt ein Ehepaar als Produktionseinheit, in der beide Ehepartner auf bestimmte Tätigkeiten spezialisiert waren (der Mann meist auf die Erwerbstätigkeit, die Frau meist auf den Haushalt), um so den grösstmöglichen Nutzen für die Familie zu erzielen (Becker 1981). Je spezialisierter das Ehepaar, desto grösser der Nutzen für alle und desto stabiler die Ehe. Waren beide erwerbstätig, was lange Zeit eher der Fall war, wenn eine Frau besser ausgebildet war, hatte dies einen negativen Effekt auf die Ehe, weil der gegenseitige Nutzen geringer ausfiel. In der heutigen Zeit mit dem weit verbreiteten Doppelverdiener-Modell gilt dieses Bild der Ehe gemäss Isen und Stevenson (2010) jedoch als veraltet. Heutzutage stehen nach Ansicht der Autoren der geteilte Konsum und die gemeinsam genutzte Freizeit im Vordergrund ("hedonic marriage"). Bei diesem Modell profitieren diejenigen Paare, bei denen beide einen Lohn erwirtschaften ("Income-Pooling"). Je höher der gemeinsame Lohn, desto höher der Lebensstandard, desto weniger finanzielle Schwierigkeiten, desto besser verteilt ist das Risiko einer allfälligen Arbeitslosigkeit und desto stabiler die Ehe. Somit sind gemäss den Autoren heute Ehen, in denen beide gut gebildet sind und gut verdienen, am stabilsten, da diese Ehepartner aus dem Income-Pooling am meisten profitieren. Andererseits könnte auch dafür argumentiert werden, dass die Stabilität der Ehe daher rührt, dass solche Ehepartner am stärksten voneinander abhängig sind, da sie alleine mit dem eigenen Lohn ihren Lebensstandard, so wie ihn das Income-Pooling ermöglicht, nicht weiterführen könnten.

Ein ähnliches Argument bringt Chen (2012) vor. Er weist darauf hin, dass höher gebildete Frauen tendenziell eine liberalere Geschlechterideologie als niedriger Gebildete vertreten. Er verweist auf Studien, die zeigen, dass Frauen mit egalitärerem Verständnis der Geschlechterrollen weniger zufrieden in ihrer Ehe sind und 
in der Folge ein höheres Scheidungsrisiko tragen. Der Autor führt Studien an, die einen Trend in Richtung Geschlechterrollen-Egalitarismus über verschiedene Länder hinweg aufzeigen (z. B. Haller et al. 2000). Werden die Ideale der egalitären Geschlechterbeziehung stärker akzeptiert, so Chen (2012), dann sind höher gebildete Frauen weniger Stress und Konflikten in der Familie ausgesetzt als vorher. Als Folge gewinnen ihre Ehen an Stabilität. Deshalb geht auch er davon aus, dass die bildungsspezifischen Unterschiede im Scheidungsverhalten von Frauen schwächer werden oder ganz verschwinden.

\subsection{Kosten-Nutzen-Modell}

Zusammenfassend kann gesagt werden, dass sich verändernde Kontextfaktoren wie eine Liberalisierung des Scheidungsrechts, eine Zunahme der Erwerbsbeteiligung der Frauen und ein Ausbau der wohlfahrtsstaatlichen Leistungen, der geringere Einfluss der Kirche und eine Verbreitung und Akzeptanz neuer Lebensformen die Scheidungskosten reduzieren, sodass das einst innovative Verhalten - eine Scheidung -, welches sich früher nur besser gebildete Frauen leisten konnten, in den letzten Jahrzehnten vor allem von niedriger gebildeten Frauen übernommen wurde. Zudem hat durch die Entwicklung hin zu einem egalitären Genderregime und durch die Transformation des Ehemodells von der Spezialisierung hin zur Symmetrie bzw. von der Produktion hin zum Konsum der Nutzen einer Ehe für Frauen mit hoher Bildung zugenommen, sodass sich diese weniger oft scheiden lassen. Als Konsequenz ergibt sich aus diesen beiden Entwicklungen, dass die Scheidungswahrscheinlichkeit bei Frauen mit höherer Bildung ab- und jene bei Frauen mit niedrigerer Bildung zugenommen hat, sodass sich der Zusammenhang zwischen dem Bildungsniveau der Frauen und dem Scheidungsverhalten über die Zeit hinweg abgeschwächt hat, anschliessend verschwunden ist und sich letztlich ins Gegenteil verkehrt hat.

\section{Schweizerischer Kontext 1950-2010}

Die im vorangegangenen Kapitel angeführten Kontextfaktoren, welche die Scheidungskosten bzw. den Nutzen einer Ehe für gebildete Frauen beeinflussen, werden für die Beschreibung des Kontexts in der Schweiz zwischen 1952 und 2013 und für die Formulierung der Hypothese herangezogen.

\subsection{Liberalisierung des Scheidungsrechts}

Zu Beginn der zweiten Hälfte des 20. Jahrhunderts war die Schweiz dem sogenannten "weak fault regime" zuzuordnen. Dieses zeichnet sich dadurch aus, dass das Scheidungsrecht erweiterbare, nicht-spezifische Scheidungsgründe anführt, welche

5 Smith unterscheidet neben dem "weak fault regime" noch das "fault regime" und das "unilateral regime". 
flexibel eine grosse Bandbreite von Vergehen umfassen, möglicherweise gar solche geringfügigen Charakters (Smith 2002). In der Schweiz war eine Scheidung aufgrund des unwiederbringlichen Scheiterns einer Ehe infolge einer komplexen Vielzahl von Gründen möglich. Somit konnte man sich gegen den Willen des Ehepartners scheiden lassen, auch wenn er oder sie sich kein ernsthaftes Vergehen hat zuschulden kommen lassen. Es gab zudem die Möglichkeit, eine Scheidung auf Basis einer dreijährigen Trennung einzuleiten; von dieser Option wurde jedoch weniger Gebrauch gemacht, da sie mehr Zeit kostete und die Scheidung abgelehnt werden konnte, wenn ein unschuldiger Ehepartner damit nicht einverstanden war (Smith 2002).

Im Jahr 2000 wurde das schweizerische Scheidungsrecht einer umfangreichen Revision unterzogen. Es gab zwei wichtige Änderungen: Erstens wurde das Verschuldensprinzip bei Unzumutbarkeit aufgehoben und zweitens die Scheidung auf gemeinsames Begehren eingeführt (BFS 2009). Im Jahr 2004 wurde zudem die Trennungsfrist bei Scheidung auf Klage eines Ehegatten von vier auf zwei Jahre gesenkt (BFS 2009). Somit gilt in der Schweiz seit dem Jahr 2000 das unilaterale Scheidungsregime (Gonzalez und Viitanen 2009), was bedeutet, dass die Hürden für eine Scheidung gesunken sind.

\subsection{Steigende Arbeitsmarktbeteiligung von Frauen}

Zwischen 1970 und 2000 schnellte die durchschnittliche Erwerbsquote der Schweizer Frauen im Haupterwerbsalter ( 25 bis 54 Jahre) von $40 \%$ auf $78 \%$ hoch (BFS 2005). Im Jahr 2010 waren es $86 \%$, was die Schweiz diesbezüglich zum Spitzenreiter innerhalb der europäischen Länder macht. Dieser Wandel ist vor allem auf einen Anstieg der Arbeitsmarktpartizipation von verheirateten Frauen und Müttern zurückzuführen (Charles 2005). Sie arbeiten im Vergleich mit Frauen aus anderen europäischen Ländern jedoch überdurchschnittlich häufig Teilzeit (Thoenen 2010).

Die Arbeitsmarktpartizipation ist in der Schweiz für den Grad an ökonomischer Unabhängigkeit einer Frau entscheidend, denn das Sozialversicherungssystem ist stark erwerbszentriert, und unbezahlte Arbeit wird nach wie vor sozialrechtlich nur ungenügend vergütet (Stutz und Knupfer 2012). So sind etwa im Scheidungsfall besonders Mütter aus einem traditionellen Familienmodell von Armut betroffen (Masia und Budowski 2009).

\subsection{Weiterhin tiefe Sozialleistungen für den Sektor «Familie/Kinder»}

Im Jahr 1995 machten die Sozialleistungen für den Sektor «Familie/Kinder» einen Anteil von $1.1 \%$ des Bruttoinlandproduktes der Schweiz aus. ${ }^{6}$ Der diesbezügliche Durchschnitt der EU-15 lag damals bei 2.1\%. Im Jahr 2011 stieg in der Schweiz der Anteil auf 1.3\%. Auch die durchschnittlichen Ausgaben der EU-15 nahmen leicht zu (2.3\%) und liegen somit sowohl im Jahr 1995 wie auch im Jahr 2011 um

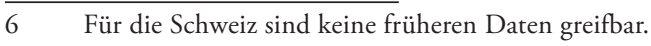


1\%-Punkt höher (BFS 2008). Die Sozialleistungen für diesen Sektor sind somit minim angestiegen und im Vergleich mit anderen europäischen Ländern nach wie vor tief.

\subsection{Abnahme der Religiosität}

Im Verlauf der zweiten Hälfte des 20. Jahrhunderts nahm die Religiosität in der Schweiz ab. Waren im Jahr 1950 56.3\% der Schweizer Bevölkerung evangelischreformiert und 41.5\% römisch-katholisch, waren es im Jahr 2012 noch $26.9 \%$ bzw. 38.2\% (BFS 2015a). Der Anteil der Konfessionslosen stieg von 0.5\% im Jahr 1960 auf 21.4\% im Jahr 2012 (BFS 2015a). Aber nicht nur der Anteil derer, die einer der beiden grössten Kirchen angehören, ist gesunken, auch der Anteil der regelmässigen Kirchgänger nahm ab. Waren es 1989 noch 41\%, sind es im Jahr 1996 noch 24\% (Nicolet und Tresch 2009).

Zudem pocht heutzutage nur noch eine kleine Minderheit auf die strikte Einhaltung der Lehre der katholischen Kirche mit ihrer strengen Disziplin. So ist eine Mehrheit der Seelsorger und Gläubiger gegen den Ausschluss wiederverheirateter Geschiedener von den Sakramenten. Denn die Gründe für das Zerbrechen von Ehen seien so vielfältig, dass die "pauschale Bestrafung durch die Kirche bei einer Wiederheirat nicht mehr akzeptiert» werde (Hehli 2015).

\subsection{Verbreitung neuer Lebensformen}

Seit 1970 sind die Ehepaarhaushalte mit Kindern stark rückläufig. Machte deren Anteil im Jahr 1970 noch mehr als 60\% aus, leben heute weniger als die Hälfte in dieser Haushaltsform (Fux 2005). Ein Vergleich sämtlicher Haushalte im Jahr 2000 zeigt, dass weniger Ehepaarhaushalte mit Kindern als Einpersonenhaushalte zu verzeichnen sind und etwa gleich viele wie Ehepaarhaushalte ohne Kinder. Zudem gibt es im Jahr 2000 im Vergleich zu 1970 markant mehr Frauen, die ganz auf Mutterschaft verzichten. ${ }^{7}$ Auch die Anzahl Personen, die unverheiratet im selben Haushalt leben, ist stark gestiegen. Zählte man im Jahr 198038129 Personen, waren es im Jahr 2000 bereits deren 130196 (Fux 2005). Den Grund dafür sieht Fux (2005) unter anderem darin, dass es in der Schweiz gesellschaftlich immer akzeptierter ist, als unverheiratetes Paar Kinder zu haben. Die gesellschaftlichen Zwänge, die nicht verheiratete Paare mit Kindern erfahren, haben zwischen 1970 und 2000 abgenommen; die Lebensformen haben sich generell diversifiziert.

\subsection{Nach wie vor konservatives Genderregime}

Die Schweiz ist weiterhin geprägt von einem konservativen Genderregime. Dieses ist durch eine relativ passive Sozialpolitik und die Erhaltung von traditionellen

$7 \quad$ Im Jahr 2000 waren 23\% der 35-44-jährigen Frauen kinderlos. Bei den 45-54-Jährigen waren es 19\% und bei den 55-64-Jährigen 15\% (BFS 2015c). 
familiären Bindungen und Normen charakterisiert. Die Familienpolitik in der Schweiz unterstützt zudem primär das männliche Ernährer-Familienmodell. Es wurden denn auch nur wenige politische Massnahmen getroffen, um die traditionelle Arbeitsteilung aufzubrechen und eine grössere finanzielle Unabhängigkeit der Frauen zu fördern (Nordenmark 2013).

\subsection{Symmetrischere Rollenverteilung in der Ehe}

Trotz eines konservativen Genderregimes hat die steigende Teilzeit-Erwerbsbeteiligung der Mütter dazu geführt, dass im Unterschied zu 1970, als das traditionelle bürgerliche Familienmodell (Mann arbeitet Vollzeit, Frau ist nicht erwerbstätig) am weitesten verbreitet war, im Jahr 2000 nun das moderne bürgerliche Familienmodell (Mann arbeitet Vollzeit, Frau Teilzeit) vorherrscht. Das egalitär-familienbezogene Modell (beide Elternteile arbeiten Teilzeit) und das egalitär-erwerbsbezogene Modell (beide Elternteile arbeiten Vollzeit) sind im Vergleich mit dem modernen bürgerlichen Modell hingegen nicht weit verbreitet (BFS 2015b). Somit ist "Income-Pooling" heutzutage zwar Standard, und eine Entwicklung weg von der spezialisierten hin zu einer mehr symmetrischen Rollenverteilung hat begonnen. Jedoch übernimmt nach wie vor meist die Frau die Hauptverantwortung für Haus- und Familienarbeit (Pfau-Effinger 2005).

Zusammenfassend kann gesagt werden, dass sich im Beobachtungszeitraum dieser Studie vor allem jene Kontextfaktoren stark verändert haben, die die Scheidungskosten beeinflussen: Das Scheidungsrecht wurde im Jahr 2000 liberalisiert, die Erwerbsbeteiligung der Frauen nahm rasant zu, was mit grösserer finanzieller Unabhängigkeit einherging, die Religiosität der Bevölkerung nahm ab und somit auch der Einfluss der Kirche, auch haben sich neue Lebensformen etabliert, die mittlerweile akzeptiert sind - allesamt Faktoren, die dazu beitragen, dass die Scheidungskosten sinken. Einzig die wohlfahrtsstaatlichen Leistungen stagnierten während der untersuchten Zeitperiode. Bei jenen Kontextfaktoren, die den Nutzen einer Ehe für gut ausgebildete Frauen erhöhen, zeigen sich hingegen nur geringfügige Veränderungen: Es dominiert in der Schweiz nach wie vor ein konservatives Genderregime und die Familienpolitik ist immer noch stark auf das Ein-ErnährerModell ausgerichtet. Zwar ist aufgrund der zunehmenden Erwerbstätigkeit der Frauen das Doppelverdiener-Modell seit den 2000er-Jahren stärker verbreitet als das Ein-Ernährer-Modell (BFS 2015b), jedoch arbeiten die Frauen in der Schweiz im Vergleich mit Frauen aus anderen europäischen Ländern überdurchschnittlich häufig Teilzeit und übernehmen nach wie vor den grössten Anteil an Hausarbeit und Kinderbetreuung (Pfau-Effinger 2005). Und die egalitären Familienmodelle (beide Elternpaare arbeiten Teil- bzw. Vollzeit) sind immer noch stark untervertreten (BFS 2015b). Somit kann davon ausgegangen werden, dass der Nutzen einer Ehe für besser gebildete Frauen im Beobachtungszeitraum dieser Studie nur geringfügig zugenommen hat. Aufgrund der gesunkenen Scheidungskosten und den nur 
geringfügig gestiegenen Nutzen einer Ehe für besser gebildete Frauen kann davon ausgegangen werden, dass sich der Zusammenhang zwischen dem Bildungsniveau der Frau und dem Scheidungsverhalten in der Schweiz zwischen 1952 und 2013 abgeschwächt, sich jedoch (noch) nicht ins Gegenteil verkehrt hat.

\section{Daten, Variablen und Methoden}

\subsection{Datensatz}

Die seit 2010 neu jährlich durchgeführte Volkszählung in der Schweiz umfasst vier Elemente: 1) die Registererhebung, welche auf der Basis bestehender Daten grundlegende Informationen zur gesamten Bevölkerung liefert, 2) die Strukturerhebung, bei der 200000 Personen mit schriftlichem oder Online-Fragebogen befragt werden, 3) die thematische Erhebung, bei der jährlich rotierend vier Themen bei einer Stichprobe von 10000 bzw. 40000 Personen telefonisch interviewt werden und 4) der Omnibus, eine telefonische Mehrthemenbefragung bei 3000 Personen.

Die für die Analyse verwendeten Daten stammen aus der thematischen Erhebung von 2013, welche sich einem Fragenkomplex rund um «Familie und Generationen» widmete. Die Gesamtheit sind alle Personen der ständigen Wohnbevölkerung in Privathaushalten zwischen 15 und 79 Jahren (Stichtag 1. Januar 2013). Nach dem Zufallsprinzip wurden aus dem Stichprobenregister des Bundesamts für Statistik 34818 Personen ausgewählt. 17288 Personen (50\%) haben letztlich an der Erhebung teilgenommen. Davon sind 53\% weiblich und 47\% männlich. Die Personen wurden einerseits telefonisch befragt und andererseits gebeten, einen ergänzenden Online- bzw. Papierfragebogen auszufüllen. Die hier verwendeten Daten stammen aus der computerunterstützten Telefonbefragung.

\subsection{Stichprobe}

Der Datensatz enthält Angaben zu 4522 in der Schweiz geborenen Frauen, die 2012, ein Jahr vor dem Befragungsjahr, mindestens einmal verheiratet waren. ${ }^{8}$ Die Analyse beschränkt sich auf Erst-Ehen, weil die Faktoren, die Zweit- und DrittEhen beeinflussen, andere sind als jene bei Erst-Ehen (Martin und Bumpass 1989).

Von 164 Frauen ist das Heiratsjahr, von 39 das Scheidungsjahr und von 8 das Jahr der Verwitwung nicht bekannt. Bei 2 Frauen ist zudem unklar, ob ihre erste Ehe mit einer Scheidung oder dem Tod des Partners geendet hat. Bei 176 Frauen fehlen Angaben zu mindestens einer unabhängigen Variablen, wobei die höchste Anzahl fehlender Angaben beim Bildungsniveau des Partners zu verzeichnen ist, nämlich 113. Sie alle wurden aus der Analyse ausgeschlossen. Es wurden zudem nur diejenigen Frauen berücksichtigt, bei deren Erst-Ehe der jüngere Ehepartner

8 Diejenigen Frauen, die im Jahr 2013 geheiratet haben, wurden aus der Analyse ausgeschlossen, da sie sich in der verbleibenden Zeit bis zur Umfrage gar nicht haben scheiden lassen können. 
mindestens 18 und höchstens 50 Jahre alt war, wodurch sich eine Stichprobe aus 4118 Frauen ergab.

\subsection{Methodenwahl}

742 der 4118 Frauen (18\%) haben im Beobachtungszeitraum eine Scheidung erlebt. Die geeignete Methode für die Auswertung dieser zum Teil rechtszensurierten Daten ist die Ereignisanalyse. Die Wahl fiel auf die Discrete-Time-Event-Analyse, da mit ihr im Unterschied zu Continuous-Time-Modellen besser simultan verlaufende Events ${ }^{9}$ analysiert werden können und sie zudem häufig in Studien zum Scheidungsverhalten angewendet wird. Sie berechnet in Abhängigkeit von ausgewählten Kontrollvariablen die konditionale Wahrscheinlichkeit, das Event (in diesem Fall eine Scheidung) zum Zeitpunkt t zu erleben, vorausgesetzt, dass es zum Zeitpunkt $\mathrm{t}-1$ noch nicht stattgefunden hat. Zur Berechnung wird eine logistische Regression verwendet (mit STATA 14 Software). ${ }^{10}$

Um die Daten für eine Discrete-Time-Event-Analyse nutzbar zu machen, müssen sie in Personen-Jahre als Analyseeinheit umorganisiert werden, wobei jede Frau mehrere Personen-Jahre zum Datensatz beiträgt: Es beginnt mit dem Heiratsjahr und endet mit dem Scheidungsjahr ${ }^{11}$, dem Todesjahr des Ehepartners ${ }^{12}$ oder, falls die Ehe bis zum Zeitpunkt der Umfrage intakt war, im Jahr 2013.

\subsection{Variablen}

Die abhängige Variable ist die Scheidung, welche mit einer 1 codiert wurde, wenn die Ehe in einem bestimmten Personen-Jahr geschieden wurde, und mit einer 0, falls nicht. Die zentrale unabhängige Variable ist der zum Befragungszeitpunkt höchste erworbene Bildungsabschluss der Frauen. Obwohl dieser zeitunabhängige Indikator nicht optimal ist, wird er in Studien zum Scheidungsverhalten oft herangezogen (z. B. Härkönen und Dronkers 2006), weil häufig, wie auch in diesem Fall, Angaben zur Bildungsbiografie fehlen.

Der höchste erworbene Bildungsabschluss der Frauen wurde in einer dreistufigen Variablen gemessen und orientiert sich an den für das Schweizer Bildungssystem gängigen Stufen Sekundarbildung 1 (obligatorische Schule), Sekundarbildung 2 (Berufslehre oder Mittelschule) und Tertiärbildung (Höhere Fach- und Berufsaus-

$9 \quad$ Es sind nur die Kalenderjahre bekannt, in denen die Heirat, die Scheidung bzw. die Verwitwung stattfand. Aus diesem Grund sind viele simultane Events zu verzeichnen.

10 Die logistischen Regressionen wurden, wie Singer und Willett (2003) anführen, ohne ClusterFunktion berechnet. Es zeigten sich bezüglich der Resultate jedoch keine relevanten Unterschiede, wenn die Cluster-Funktion berücksichtigt wurde. Es wurde zudem getestet, ob die Wahl der Linkfunktion in den statistischen Modellen (logit oder complementary log-log) unterschiedliche Ergebnisse liefert. Die Resultate sind bei beiden Linkfunktionen sehr ähnlich.

11 Es werden nur offizielle Scheidungen gezählt. Waren die Ehepaare zum Zeitpunkt der Befragung noch in Scheidung, galt die Ehe als nicht geschieden und somit noch als intakt.

12335 Ehen endeten mit dem Tod eines Ehepartners. Diese wurden zum Todeszeitpunkt für die Untersuchung zensuriert. 
Tabelle 1 Deskriptive Statistiken der unabhängigen Variablen

\begin{tabular}{|c|c|}
\hline Variable & Kennzahl \\
\hline \multicolumn{2}{|l|}{ Bildung der Frau (in Prozent) } \\
\hline tief & 20.9 \\
\hline mittel & 64.3 \\
\hline hoch & 14.8 \\
\hline \multicolumn{2}{|l|}{ Bildung des Partners (in Prozent) } \\
\hline & 9.9 \\
\hline mittel & 60.0 \\
\hline hoch & 30.1 \\
\hline Ehedauer (in Jahren, arithmetisches Mittel) & $\begin{array}{r}17.6 \\
(12.7)\end{array}$ \\
\hline Beobachtungsjahr (in Jahren, arithmetisches Mittel) & $\begin{array}{r}43.0 \\
(13.3)\end{array}$ \\
\hline \multirow[t]{2}{*}{ Heiratsalter des jüngeren Ehepartners (in Jahren, arithmetisches Mittel) } & 24.6 \\
\hline & $(4.2)$ \\
\hline Voreheliches Zusammenleben des Paares (in Prozent) & 35.0 \\
\hline \multicolumn{2}{|l|}{ Anzahl Kinder (in Prozent) } \\
\hline kein Kind & 16.3 \\
\hline 1 Kind & 18.3 \\
\hline 2 Kinder & 40.2 \\
\hline 3 Kinder und mehr & 25.2 \\
\hline Voreheliche(s) Kind(er) des Paares (in Prozent) & 4.2 \\
\hline Scheidung der Eltern (in Prozent) & 8.0 \\
\hline Nach der Scheidungsrechtsrevision (in Prozent) & 40.8 \\
\hline \multicolumn{2}{|l|}{ Heiratskohorten (in Prozent) } \\
\hline $1952-1959$ & 6.8 \\
\hline $1960-1969$ & 27.5 \\
\hline $1970-1979$ & 23.7 \\
\hline 1980-1989 & 20.5 \\
\hline 1990-1999 & 14.9 \\
\hline $2000-2012$ & 6.6 \\
\hline
\end{tabular}

Anmerkungen: Personen-Jahre; Standardabweichung in Klammern. Die Variable "Beobachtungsjahr" wurde umcodiert, sodass das erste Beobachtungsjahr (1952) 1 entspricht.

Quelle: Erhebung zu Familien und Generationen 2013 (BFS), eigene Berechnungen.

bildung, Universität oder Fachhochschule). ${ }^{13}$ Personen ohne Abschluss wurden der Sekundarstufe 1 zugeordnet.

Folgende in Studien zum Scheidungsverhalten gängige Kontrollvariablen wurden berücksichtigt: 1) die Ehedauer in Jahren, welche sowohl linear wie auch

13 Die hier verwendete Dreigliederung entspricht den Stufen 0-II, III und IV bzw. V und VI der International Standard Classification of Education (ISCED 97). 
im Quadrat in die Modelle aufgenommen wird $\left.{ }^{14}, 2\right)$ das Beobachtungsjahr ${ }^{15}$, 3) der höchste erworbene Bildungsabschluss des Ehepartners (gleich codiert wie bei der Frau $)^{16}$, 4) das Heiratsalter des jüngeren Ehepartners in Jahren, 5) voreheliches Zusammenleben ( $\mathrm{Ja}=1$; Nein $=0), 6)$ ein voreheliches Kind, wobei dies ein gemeinsames Kind sein kann wie auch eines aus einer früheren Beziehung $(\mathrm{Ja}=1$; Nein $=0)$, 7) die Anzahl leiblicher Kinder der Frau (kein Kind =1; 1 Kind = 2; 2 Kinder =3; 3 Kinder und $\mathrm{mehr}=4)$ und 8) eine Scheidung der Eltern $(\mathrm{Ja}=1$; Nein =0). Zwei weitere Variablen, welche in der Analyse herangezogen wurden, sind ein Dummy für die Personen-Jahre vor bzw. nach Inkrafttreten der Scheidungsrechtsrevision im Jahr 2000 (ab dem Jahr $2000=1$; vor dem Jahr 2000 =0) und die Heiratskohorten $(1952$ bis $1959=1 ; 1960$ bis $1969=2 ; 1970$ bis $1979=3 ; 1980$ bis $1989=4 ; 1990$ bis $1999=5$; 2000 bis $2012=6$ ).

Nicht berücksichtigt wurden zwei in Studien zum Scheidungsrisiko gängige zeitabhängige Variablen: die Erwerbsbeteiligung sowie das Einkommen, da weder die Erwerbs- noch die Einkommensbiografie im Datensatz enthalten sind. Es sind lediglich das Einkommen und der Erwerbsstatus zum Zeitpunkt des Interviews also im Jahr 2013 - bekannt. ${ }^{17}$

Tabelle 1 weist die deskriptiven Angaben zu den unabhängigen Variablen aus.

\subsection{Analytisches Vorgehen}

Es werden sechs Modelle berechnet. Das erste (Modell A) stellt ein reduziertes Modell mit Bildungsniveau der Frauen, Ehedauer (linear und im Quadrat) und Beobachtungsjahr dar. Die minimale Anzahl von Kontrollvariablen dient hierbei der Einschätzung des Gesamteffekts des Bildungsniveaus. Im zweiten Modell (Modell B) werden zusätzlich die Variablen Bildungsniveau des Ehepartners, Heiratsalter des jüngeren Ehepartners, voreheliches Kind, Anzahl Kinder, voreheliches Zusammenleben und Scheidung der Eltern der Frau berücksichtigt. Einige dieser Variablen sind nicht als Kontrollvariablen im engeren Sinne zu verstehen, weil sie vom Bildungsni-

14 Die ganze Ehedauer wurde in der Analyse berücksichtigt. Die Signifikanzen des Bildungsniveaus der Frauen und der Interaktionsterme bleiben gleich, wenn man die Ehen nur bis zum 15. Ehejahr beobachtet, d. h. längere Ehen im 15. Ehejahr für die Untersuchung zensuriert.

15 Um die Interpretation der Resultate in den multivarianten Analysen zu vereinfachen, wurde das Beobachtungsjahr umcodiert, sodass das erste Beobachtungsjahr (1952) 1 entspricht.

16 Die Kontrolle des Bildungsniveaus des Partners ist wichtig, weil auch dieser sowie die Bildungshomogamie in den letzten Jahrzehnten zugenommen haben, was der Ehestabilität zuträglich ist und die Resultate ohne diesbezügliche Kontrolle somit verzerrt werden können (Schwartz und Mare 2005).

17 In den verwendeten Daten liegt die Scheidung durchschnittlich 16 Jahre zurück (früheste Scheidung 1961, späteste Scheidung: 2013). In diesen durchschnittlich 16 Jahren sind die Geschiedenen unter Umständen neue Partnerschaften eingegangen, haben wieder geheiratet, haben (weitere) Kinder bekommen, sind arbeitslos oder krank geworden, sind in den Ruhestand getreten usw. Dies sind alles Ereignisse, die sich auf das Einkommen und den Erwerbsstatus auswirken und die zum Zeitpunkt der Scheidung in vielen Fällen nicht voraussehbar waren. Aus diesen Gründen wird darauf verzichtet, das Einkommen und dem Erwerbsstatus im Jahr 2013 zur Erklärung des Scheidungsverhaltens zu einem früheren Zeitpunkt heranzuziehen. 
veau der Frauen abhängen, so z. B. das Heiratsalter. Diese Variablen werden jedoch häufig in Modellen zur Berechnung des Scheidungsverhaltens herangezogen und ermöglichen eine konservativere Schätzung des Einflusses des Bildungsniveaus von Frauen auf das Scheidungsverhalten. Das dritte Modell (Modell C) enthält zudem den Interaktionsterm zwischen dem Bildungsniveau und dem Beobachtungsjahr. Dies, um zu analysieren, ob sich der Effekt des Bildungsniveaus über die Zeit hinweg verändert hat. Im vierten Modell (Modell D) wird zusätzlich eine Dummyvariable für alle Personen-Jahre ab dem Jahr 2000, also ab dem Inkrafttreten des neuen Scheidungsrechts, eingefügt. Im fünften Modell (Modell E) wird das Beobachtungsjahr (Periodeneffekt) durch die Heiratskohorten (Kohorteneffekt) ersetzt. Und das sechste Modell (Modell F) umfasst sowohl das Beobachtungsjahr wie auch die Heiratskohorten, um zu sehen, ob der Perioden- oder der Kohorteneffekt stärker ist.

Die Modelle wurden mit dem Log-Likelihood-Ratio-Test miteinander verglichen, um zu eruieren, ob das mit mehr Variablen bestückte Modell signifikant bessere Resultate liefert, was jeweils der Fall war. Es wurden zudem die Interaktionen zwischen allen Variablen und der Ehedauer getestet: Lediglich die Anzahl Kinder verletzt die Proportionalitätsannahme, weshalb ab dem zweiten Modell zudem noch eine Interaktion zwischen der Anzahl Kinder und der Ehedauer berücksichtigt wird.

Die geschätzten Koeffizienten der Interaktionsvariablen im Modell C erlauben keine direkte Interpretation. Der Koeffizient von X (im vorliegenden Fall: das Bildungsniveau der Frauen) fängt in nicht-linearen Modellen nur die Wirkung von X auf Y (hier: das Scheidungsrisiko) ein, wenn Z (hier: das Beobachtungsjahr) gleich null ist (Brambor et al. 2005). Weiter fängt $Z$ nur die Wirkung von $Z$ auf $Y$ ein, wenn $X$ gleich null ist. Im vorliegenden Fall sind jedoch weder $Z$ noch $X$ gleich null. Wie Ai und Norton (2003) zeigen, kann nicht einmal aufgrund der Höhe und der Signifikanz des Koeffizienten der Interaktionsvariablen gefolgert werden, dass X eine konditionale Wirkung auf Y hat. Um die Koeffizienten der Interaktionsvariablen zu interpretieren, eignen sich gemäss Williams (2012) APRs ("Adjusted Predictions at Representative Values") und MERs ("Marginal Effects at Representative Values"). Im vorliegenden Fall geben die APRs für jedes Kalenderjahr an, wie hoch die Wahrscheinlichkeit einer Scheidung für die drei Bildungsgruppen ist. Die MERs wiederum zeigen hier für jedes Kalenderjahr auf, um wie viele Prozentpunkte sich die Wahrscheinlichkeit einer Scheidung für die Gruppe der Frauen mit einer mittleren bzw. hohen Bildung im Vergleich zur Referenzgruppe (der Gruppe der Frauen mit einer tiefen Bildung) unterscheidet (Williams 2012).

\section{Ergebnisse}

In Tabelle 2 sind die Ergebnisse der oben erwähnten Modelle aufgeführt. Zuerst werden die Effekte der Kontrollvariablen besprochen. 


\subsection{Effekte der Kontrollvariablen}

In Modell B zeigt sich eine kurvilineare Beziehung zwischen der Ehedauer und dem Scheidungsverhalten; das heisst, dass das Scheidungsrisiko in den ersten Ehejahren steigt und dann im Verlauf der Ehe wieder abnimmt. Über die Beobachtungsjahre hinweg gesehen nimmt das Scheidungsrisiko hingegen zu.

Das Heiratsalter des jüngeren Ehepartners weist einen signifikant negativen Zusammenhang mit dem Scheidungsverhalten auf: Je tiefer das Heiratsalter, desto häufiger wird geschieden. Gründe hierfür könnten sein, dass jüngere Personen weniger reif sind, unvorbereiteter weitreichende Entscheidungen fällen und sich für eine kürzere Zeitspanne auf dem «Heiratsmarkt» bewegen, was die Gefahr einer schlechten Partnerwahl erhöht (South 1995). Jüngere haben zudem oft eine grössere Auswahl an Partnern, wodurch die Hürden für eine Scheidung sinken (Lyngstad und Jalovaara 2010).

Voreheliches Zusammenleben hingegen wirkt sich positiv aus: Diejenigen Paare, die vor der Heirat bereits zusammengelebt haben, weisen ein höheres Scheidungsrisiko auf als solche, die erst nach der Hochzeit zusammengezogen sind. Dieses Resultat lässt sich primär durch einen Selektionseffekt erklären. Die bereits vor der Ehe zusammenlebenden Personen vertreten liberalere Werte, weshalb sie sich auch häufiger scheiden lassen (Härkönen 2005).

Ein voreheliches Kind erhöht das Scheidungsrisiko, da Frauen, die vor der Heirat schwanger werden oder ein Kind auf die Welt bringen, oft übereilt heiraten und weniger Zeit haben, eine stabile Beziehung aufzubauen (Hoem und Hoem 1992).

Alle Frauen mit mindestens einem Kind haben ein tieferes Scheidungsrisiko als jene ohne Kinder. Dies kann einerseits damit erklärt werden, dass Kinder ehespezifisches Kapital darstellen. Dies bedeutet, dass der Nutzen vom Kinderhaben grösser ist, wenn die Eltern zusammenleben, als wenn sie getrennt sind. Andererseits kann es sich hier auch um einen Selektionseffekt handeln: Ehepaare, die ihre Ehe als stabil erachten, entscheiden sich häufiger für ein Kind als jene, welche ihrer Ehe weniger Chancen geben (Lyngstad und Jalovaara 2010). Zudem zeigt sich, dass das Scheidungsrisiko mit jedem zusätzlichen Kind abnimmt, da das ehespezifische Kapital immer grösser und somit die Hürde für eine Scheidung höher wird.

Hat eine Frau die Scheidung ihrer Eltern erlebt, so erhöht sich das Scheidungsrisiko. Dieses Phänomen wird intergenerationale Transmission der Scheidung genannt (Amato 1996). Schliesslich ist anzumerken, dass der Einfluss des Bildungsniveaus des Partners insignifikant ist, was überrascht, da sich in anderen Studien meist zeigt, dass ein hohes Bildungsniveau des Mannes das Scheidungsrisiko senkt (z. B. Poortman und Kalmijn 2002). ${ }^{18}$

18 Alternativ wurde zum Bildungsstand des Partners die Bildungshomogamie in die Analyse aufgenommen, welche basierend auf der Dreigliederung des Bildungsniveaus für Ehefrau und -mann wie folgt kodiert wurde: $1=$ Ehemann hat höheres Bildungsniveau als Ehefrau, $2=$ beide haben gleiches Bildungsniveau, 3 = Ehefrau hat höheres Bildungsniveau als Ehemann. Es zeigten sich keine signifikanten Unterschiede im Scheidungsrisiko. 
Tabelle 2 Determinanten des Scheidungsrisikos

\begin{tabular}{|c|c|c|c|c|c|c|}
\hline Variablen & Modell A & Modell B & Modell C & Modell D & Modell E & Modell F \\
\hline \multicolumn{7}{|l|}{$\begin{array}{l}\text { Bildungsniveau der Frau (Ref. tiefe } \\
\text { Bildung) }\end{array}$} \\
\hline mittlere Bildung & $\begin{array}{c}0.312^{*} \\
(0.120)\end{array}$ & $\begin{array}{l}0.357^{* *} \\
(0.122)\end{array}$ & $\begin{array}{c}0.977^{*} \\
(0.442)\end{array}$ & $\begin{array}{l}0.358^{* *} \\
(0.122)\end{array}$ & $\begin{array}{l}0.357^{* *} \\
(0.123)\end{array}$ & $\begin{array}{l}0.346^{* *} \\
(0.123)\end{array}$ \\
\hline hohe Bildung & $\begin{array}{l}0.462^{* *} \\
(0.142)\end{array}$ & $\begin{array}{l}0.624^{* * *} \\
(0.149)\end{array}$ & $\begin{array}{l}2.277^{* * *} \\
(0.534)\end{array}$ & $\begin{array}{l}0.625^{* * *} \\
(0.149)\end{array}$ & $\begin{array}{l}0.636^{* * *} \\
(0.149)\end{array}$ & $\begin{array}{l}0.616^{* * *} \\
(0.149)\end{array}$ \\
\hline Ehedauer (linear) & $\begin{array}{l}0.106^{* * *} \\
(0.013)\end{array}$ & $\begin{array}{l}0.156^{* * *} \\
(0.016)\end{array}$ & $\begin{array}{l}0.157^{* * *} \\
(0.016)\end{array}$ & $\begin{array}{l}0.156^{* * *} \\
(0.016)\end{array}$ & $\begin{array}{l}0.178^{* * *} \\
(0.017)\end{array}$ & $\begin{array}{l}0.150 * * * \\
(0.020)\end{array}$ \\
\hline Ehedauer im Quadrat & $\begin{array}{l}-0.003^{* * *} \\
(0.000)\end{array}$ & $\begin{array}{l}-0.006^{* * *} \\
(0.000)\end{array}$ & $\begin{array}{l}-0.006^{* * *} \\
(0.000)\end{array}$ & $\begin{array}{l}-0.006^{* * *} \\
(0.000)\end{array}$ & $\begin{array}{l}-0.006^{* * *} \\
(0.000)\end{array}$ & $\begin{array}{l}-0.006^{* * *} \\
(0.000)\end{array}$ \\
\hline Beobachtungsjahr & $\begin{array}{l}0.026^{* * *} \\
(0.003)\end{array}$ & $\begin{array}{l}0.031 * * * \\
(0.003)\end{array}$ & $\begin{array}{l}0.048^{* * *} \\
(0.009)\end{array}$ & $\begin{array}{l}0.028^{* * *} \\
(0.005)\end{array}$ & & $\begin{array}{c}0.031 * \\
(0.013)\end{array}$ \\
\hline Heiratsalter des jüngeren Ehepartners & & $\begin{array}{l}-0.115^{* * *} \\
(0.011)\end{array}$ & $\begin{array}{c}-0.111^{* * *} \\
(0.011)\end{array}$ & $\begin{array}{l}-0.115^{* * *} \\
(0.011)\end{array}$ & $\begin{array}{c}-0.109 * * * \\
(0.011)\end{array}$ & $\begin{array}{l}-0.113^{* * *} \\
(0.011)\end{array}$ \\
\hline Voreheliches Zusammenleben & & $\begin{array}{l}0.475^{* * *} \\
(0.086)\end{array}$ & $\begin{array}{l}0.464^{* * *} \\
(0.086)\end{array}$ & $\begin{array}{l}0.476^{* * *} \\
(0.086)\end{array}$ & $\begin{array}{l}0.465^{* * *} \\
(0.087)\end{array}$ & $\begin{array}{l}0.449 * * * \\
(0.087)\end{array}$ \\
\hline Voreheliches Kind & & $\begin{array}{l}0.730^{* * *} \\
(0.163)\end{array}$ & $\begin{array}{l}0.698^{* * *} \\
(0.164)\end{array}$ & $\begin{array}{l}0.731^{* * *} \\
(0.163)\end{array}$ & $\begin{array}{l}0.742^{* * *} \\
(0.163)\end{array}$ & $\begin{array}{l}0.735^{* * *} \\
(0.163)\end{array}$ \\
\hline \multicolumn{7}{|l|}{ Anzahl Kinder (Ref. kein Kind) } \\
\hline 1 Kind & & $\begin{array}{c}-1.112^{* * *} \\
(0.195)\end{array}$ & $\begin{array}{l}-1.089 * * * \\
(0.195)\end{array}$ & $\begin{array}{l}-1.115^{* * *} \\
(0.195)\end{array}$ & $\begin{array}{l}-1.095^{* * *} \\
(0.195)\end{array}$ & $\begin{array}{c}-1.098^{* * *} \\
(0.195)\end{array}$ \\
\hline 2 Kinder & & $\begin{array}{c}-2.064^{* * *} \\
(0.226)\end{array}$ & $\begin{array}{l}-2.040 * * * \\
(0.226)\end{array}$ & $\begin{array}{l}-2.067^{* * *} \\
(0.226)\end{array}$ & $\begin{array}{l}-2.046^{* * *} \\
(0.226)\end{array}$ & $\begin{array}{c}-2.046^{* * *} \\
(0.226)\end{array}$ \\
\hline $3+$ Kinder & & $\begin{array}{l}-3.269^{* * *} \\
(0.363)\end{array}$ & $\begin{array}{l}-3.230 * * * \\
(0.363)\end{array}$ & $\begin{array}{l}-3.227^{* * *} \\
(0.363)\end{array}$ & $\begin{array}{l}-3.251^{* * *} \\
(0.363)\end{array}$ & $\begin{array}{c}-3.254^{\star * *} \\
(0.364)\end{array}$ \\
\hline Scheidung der Eltern & & $\begin{array}{l}0.465^{* * *} \\
(0.104)\end{array}$ & $\begin{array}{l}0.469^{* * *} \\
(0.104)\end{array}$ & $\begin{array}{l}0.464^{* * *} \\
(0.104)\end{array}$ & $\begin{array}{l}0.480 * * * \\
(0.105)\end{array}$ & $\begin{array}{l}0.474^{* * *} \\
(0.105)\end{array}$ \\
\hline \multicolumn{7}{|l|}{$\begin{array}{l}\text { Bildung des Ehepartners } \\
\text { (Ref. tiefe Bildung) }\end{array}$} \\
\hline mittlere Bildung & & $\begin{array}{c}0.033 \\
(0.139)\end{array}$ & $\begin{array}{c}0.021 \\
(0.139)\end{array}$ & $\begin{array}{c}0.035 \\
(0.139)\end{array}$ & $\begin{array}{c}0.030 \\
(0.140)\end{array}$ & $\begin{array}{c}0.020 \\
(0.140)\end{array}$ \\
\hline hohe Bildung & & $\begin{array}{l}-0.086 \\
(0.154)\end{array}$ & $\begin{array}{c}-0.103 \\
(0.154)\end{array}$ & $\begin{array}{c}-0.084 \\
(0.154)\end{array}$ & $\begin{array}{c}-0.096 \\
(0.154)\end{array}$ & $\begin{array}{c}-0.098 \\
(0.154)\end{array}$ \\
\hline Scheidungsrechtsrevision im Jahr 2000 & & & & $\begin{array}{c}0.092 \\
(0.126)\end{array}$ & & \\
\hline \multicolumn{7}{|l|}{ Heiratskohorte (Ref. 1950-1959) } \\
\hline 1960-1969 & & & & & $\begin{array}{c}0.155 \\
(0.259)\end{array}$ & $\begin{array}{r}-0.093 \\
(0.279)\end{array}$ \\
\hline 1970-1979 & & & & & $\begin{array}{l}0.581^{*} \\
(0.253)\end{array}$ & $\begin{array}{c}0.050 \\
(0.337)\end{array}$ \\
\hline 1980-1989 & & & & & $\begin{array}{l}0.906^{* * *} \\
(0.255)\end{array}$ & $\begin{array}{c}0.077 \\
(0.431)\end{array}$ \\
\hline 1990-1999 & & & & & $\begin{array}{l}1.146^{* * *} \\
(0.264)\end{array}$ & $\begin{array}{c}0.039 \\
(0.534)\end{array}$ \\
\hline $2000-2012$ & & & & & $\begin{array}{l}1.290^{* * *} \\
(0.291)\end{array}$ & $\begin{array}{l}-0.121 \\
(0.660)\end{array}$ \\
\hline
\end{tabular}


Fortsetzung von Tabelle 2.

\begin{tabular}{|c|c|c|c|c|c|c|}
\hline Variablen & Modell A & Modell B & Modell C & Modell D & Modell E & Modell F \\
\hline \multicolumn{7}{|c|}{$\begin{array}{l}\text { Anzahl Kinder*Ehedauer (Ref. kein } \\
\text { Kind }{ }^{*} \text { Ehedauer) }\end{array}$} \\
\hline 1 Kind $^{*}$ Ehedauer & & $\begin{array}{l}0.056^{* * *} \\
(0.015)\end{array}$ & $\begin{array}{l}0.056^{* * *} \\
(0.015)\end{array}$ & $\begin{array}{l}0.056^{* * *} \\
(0.015)\end{array}$ & $\begin{array}{l}0.056^{* * *} \\
(0.015)\end{array}$ & $\begin{array}{l}0.056^{* * *} \\
(0.015)\end{array}$ \\
\hline 2 Kinder*Ehedauer & & $\begin{array}{l}0.085^{* * *} \\
(0.15)\end{array}$ & $\begin{array}{l}0.084^{* * *} \\
(0.015)\end{array}$ & $\begin{array}{l}0.085^{* * *} \\
(0.015)\end{array}$ & $\begin{array}{l}0.085^{* * *} \\
(0.015)\end{array}$ & $\begin{array}{l}0.084^{* * *} \\
(0.015)\end{array}$ \\
\hline $3+$ Kinder $^{*}$ Ehedauer & & $\begin{array}{l}0.121^{* * *} \\
(0.019)\end{array}$ & $\begin{array}{l}0.120^{* * *} \\
(0.019)\end{array}$ & $\begin{array}{l}0.121^{* * *} \\
(0.019)\end{array}$ & $\begin{array}{l}0.121^{* * *} \\
(0.019)\end{array}$ & $\begin{array}{l}0.120^{* * *} \\
(0.019)\end{array}$ \\
\hline $\begin{array}{l}\text { Mittlere Bildung der Frau* } \\
\text { Beobachtungsjahr }\end{array}$ & & & $\begin{array}{r}-0.015 \\
(0.009)\end{array}$ & & & \\
\hline $\begin{array}{l}\text { Hohe Bildung der Frau* } \\
\text { Beobachtungsjahr }\end{array}$ & & & $\begin{array}{l}-0.036^{* *} \\
(0.011)\end{array}$ & & & \\
\hline Konstante & $\begin{array}{l}-6.768^{* * *} \\
(0.186)\end{array}$ & $\begin{array}{l}-3.901^{* * *} \\
(0.307)\end{array}$ & $\begin{array}{l}-4.693^{* * *} \\
(0.488)\end{array}$ & $\begin{array}{l}-3.798^{* * *} \\
(0.337)\end{array}$ & $\begin{array}{l}-3.787^{* * *} \\
(0.371)\end{array}$ & $\begin{array}{l}-3.884^{* * *} \\
(0.374)\end{array}$ \\
\hline
\end{tabular}

Anmerkungen: Diskrete Ereignisanalysen, nichtstandardisierte Koeffizienten, Standardfehler in Klammern, Anzahl Ehen $=4118$, Anzahl Ereignisse $=742$, Anzahl Personen-Jahre $=103445$.

Datenbasis: Erhebung zu Familien und Generationen 2013 (BFS), eigene Berechnungen.

${ }^{*} p \leq 0.05 ;{ }^{* *} p \leq 0.01 ;{ }^{* * *} p \leq 0.001$.

Alle anderen oben angeführten Resultate stimmen mit den bisherigen Studien über die Determinanten des Scheidungsverhaltens überein (White 1990; Wagner und Weiss 2006).

\subsection{Haupteffekt: Bildungsniveau der Frauen}

An dieser Stelle soll die Aufmerksamkeit auf die Hauptvariable, das Bildungsniveau der Frauen, gelenkt werden. Im Modell A von Tabelle 2 wird ersichtlich, dass der Zusammenhang zwischen dem Bildungsniveau und dem Scheidungsverhalten positiv und signifikant ist. Es gilt somit generell: Das Scheidungsrisiko ist für Frauen mit hoher Bildung höher im Vergleich zu Frauen mit niedrigerer Bildung, und zwar etwa um das Anderthalbfache $[\exp (0.462)=1.58]$. Auch die Berücksichtigung des Heiratsalters des jüngeren Ehepartners, des vorehelichen Zusammenlebens, der Existenz eines vorehelichen Kindes, der Anzahl Kinder, der Scheidung der Eltern einer Frau und des Bildungsniveaus des Ehepartners (Modell B) ändert nichts am Resultat: Der Einfluss des Bildungsniveaus von Frauen auf das Scheidungsverhalten bleibt signifikant und positiv.

\subsection{Zeitabhängiger Effekt des Bildungsniveaus der Frauen}

In Modell C ist ersichtlich, dass die Interaktionsterme zwischen dem Bildungsniveau der Frauen und dem Beobachtungsjahr ein negatives Vorzeichen aufweisen und jener mit den beiden interagierten Variablen «hohe Bildung der Frau» und «Beob- 
achtungsjahr» signifikant ist. Der Einfluss des Bildungsniveaus der Frauen auf das Scheidungsverhalten hat sich somit zwischen 1952 und 2013 abgeschwächt, wobei diese Resultate - wie im Kapitel 4.5 beschrieben - nicht direkt, sondern anhand der Marginal Effects interpretiert werden müssen.

Schauen wir die APRs der Scheidungswahrscheinlichkeit nach Bildungsgruppe in den einzelnen Beobachtungsjahren basierend auf Modell $\mathrm{C}$ an (Abbildung 1). Es zeigt sich, dass die Wahrscheinlichkeit, eine Scheidung zu erleben, für alle Frauen auch für diejenigen mit hoher Bildung - über die Zeit hinweg zugenommen hat. Die Scheidungswahrscheinlichkeit hat für die am niedrigsten gebildeten Frauen und etwas weniger ausgeprägt auch für Frauen mit mittlerer Bildung über die Zeit hinweg überproportional zugenommen, was vor dem Hintergrund der diesen Artikel leitenden These so interpretiert werden könnte, dass die Scheidungskosten sanken, sodass sich nun vermehrt auch die niedriger Gebildeten, deren Ehen grundsätzlich belasteter und somit instabiler sind, haben scheiden lassen können. Die Scheidungswahrscheinlichkeit nimmt aber auch für Frauen mit hoher Bildung über den gesamten Beobachtungszeitraum zu, jedoch viel weniger stark. Im Rahmen der hier leitenden These könnte dies dahingehend interpretiert werden, dass aufgrund des in der Schweiz trotz der mittlerweile hohen Arbeitsmarktbeteiligung der Frauen und des stark verbreiteten Doppelverdiener-Modells nach wie vor dominierenden konservativen Genderregimes und der nur langsamen Ablösung einer spezialisierten Rollenverteilung durch ein symmetrisches Ehemodell der Nutzen einer Ehe für besser gebildete Frauen zu geringfügig gestiegen ist, als dass in der Folge die Scheidungswahrscheinlichkeit gesunken wäre.

Schaut man sich die Entwicklung der Scheidungswahrscheinlichkeit der einzelnen Bildungsgruppen genauer an ${ }^{19}$, dann zeigen sich bis ins Jahr 1999 signifikante Unterschiede: Je höher das Bildungsniveau der Frauen, desto grösser ist die Wahrscheinlichkeit, dass sie eine Scheidung erleben. Ab 2000 sind keine signifikanten Unterschiede mehr zwischen den Frauen mit mittlerer Bildung und jenen mit niedrigerer Bildung zu verzeichnen. Und ab 2004 können zwischen den drei Bildungsgruppen gar keine signifikanten Unterschiede mehr festgestellt werden. Im Jahr 2012 ist die Scheidungswahrscheinlichkeit für Frauen mit einer mittleren Bildung gar grösser (wenn auch nicht signifikant) als für jene mit hoher Bildung. Und im Jahr 2013 haben sowohl die Frauen mit einer niedrigen wie auch jene mit einer mittleren Bildung eine höhere Scheidungswahrscheinlichkeit als Frauen mit einer hohen Bildung (auch hier sind die Koeffizienten jedoch nicht signifikant).

Eine andere Darstellungsvariante stellen die MERs dar (Abbildung 2). Bei deren Betrachtung wird ersichtlich ${ }^{20}$, dass die Unterschiede hinsichtlich des Schei-

19 Die Marginal Effects (APRs) pro Bildungsgruppe und Jahr sind hier aus Platzgründen nicht in absoluten Zahlen tabellarisch aufgeführt, sondern werden nur in Abbildung 1 als Grafik ausgewiesen. 
Abbildung $1 \quad$ Scheidungsrisiko nach Bildungsniveau, 1952-2013

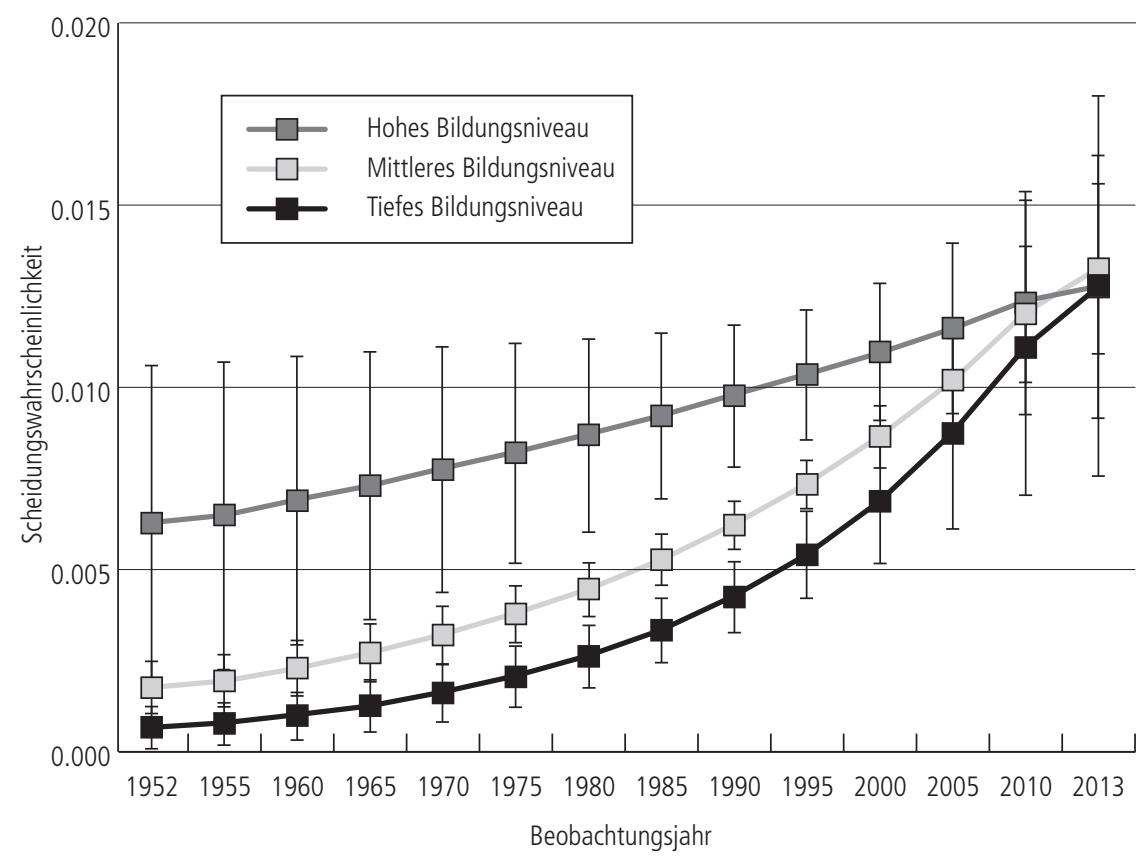

Anmerkungen: Adjusted Predictions at Representative Values.

Quelle: Erhebung zu Familien und Generationen 2013 (BFS), eigene Berechnungen.

dungsrisikos zwischen der höchsten und der niedrigsten Bildungsgruppe bis ins Jahr 1974 noch leicht zu-, danach abnehmen, was auf das ansteigende Scheidungsrisiko der Frauen mit einer tiefen Bildung zurückzuführen ist. Ab dem Jahr 2013 ist der Unterschied negativ und das Scheidungsrisiko für höher Gebildete somit kleiner als für niedrig Gebildete, jedoch werden die Differenzen - wie oben bereits erwähnt ab dem Jahr 2004 insignifikant. Die Unterschiede zwischen der mittleren und der niedrigen Bildungsgruppe nehmen bis ins Jahr 1990 leicht zu, werden dann vor allem aufgrund des ansteigenden Scheidungsrisikos der Frauen mit einer niedrigen Bildung wieder kleiner, wobei die Unterschiede ab dem Jahr 2000 insignifikant sind.

Die Tatsache, dass der Wechsel von signifikanten zu insignifikanten Unterschieden zwischen den Bildungsgruppen im Jahr 2000 erfolgt, als in der Schweiz das Scheidungsrecht revidiert wurde und die Hürden für eine Scheidung sanken, könnte als Bestätigung für Goodes Annahme interpretiert werden, dass ein vereinfachter Zugang zur Scheidung zu einer Veränderung des Zusammenhangs zwischen dem 
Abbildung 2 Unterschiede im Scheidungsrisiko zwischen den Bildungsniveaus, 1952-2013

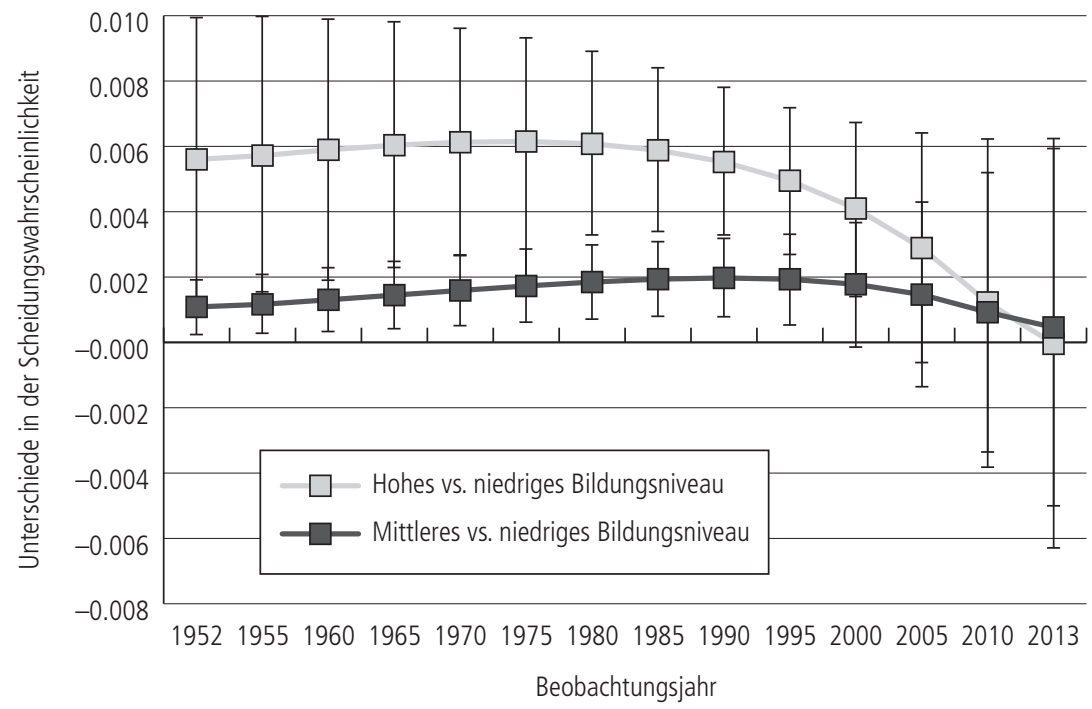

Anmerkungen: Marginal Effects at Representative Values.

Quelle: Erhebung zu Familien und Generationen 2013 (BFS), eigene Berechnungen.

Bildungsniveau der Frauen und dem Scheidungsverhalten führt. Jedoch hat der Trend, dass tiefer und niedrig gebildete Frauen sich zunehmend scheiden lassen, wie in Abbildung 1 ersichtlich, schon vor dem Jahr 2000 eingesetzt, sodass auch dafür argumentiert werden könnte, dass die Zunahme sowie die gesellschaftliche Akzeptanz von Scheidungen in der Liberalisierung des Scheidungsrechts ihren Niederschlag fand und die Scheidungsrechtsrevision somit keine Auswirkungen auf das Scheidungsverhalten hatte. Für Letzteres spricht Modell D, in welchem zusätzlich eine Dummyvariable für die Personen-Jahre ab dem Jahr 2000 (in diesem Jahr trat die Scheidungsrechtsrevision in Kraft) aufgenommen wurde. Die Dummyvariable ist insignifikant. Dieses Ergebnis stimmt mit vielen Studien überein, welche belegen, dass die Liberalisierung des Scheidungsrechts allenfalls eine kleine Zunahme an Scheidungen nach sich zieht (Härkönen 2013).

Die Analyse der bildungsspezifischen Unterschiede im Scheidungsverhalten der Frauen in der Schweiz von 1952 bis 2013 hat somit ergeben, dass - wie in der Hypothese formuliert - die Scheidungswahrscheinlichkeit vor allem für Frauen mit tiefer, aber auch, wenn auch etwas weniger ausgeprägt, für jene mit mittlerer Bildung zugenommen und für Frauen mit einer hohen Bildung (noch) nicht abgenommen 
hat. Die erstgenannte Entwicklung führt dazu, dass - wie in der Hypothese formuliert - bezüglich des Zusammenhangs zwischen dem Bildungsniveau der Frauen und dem Scheidungsverhalten eine Veränderung stattgefunden hat: Der Zusammenhang hat sich abgeschwächt und ist dann verschwunden. Er hat sich jedoch bis ins Jahr 2013 (noch) nicht ins Gegenteil verkehrt. Ein Grund dafür könnte - wird der hier leitenden These gefolgt - darin liegen, dass der Nutzen der Ehe für Frauen mit hoher Bildung (noch) zu geringfügig gestiegen ist.

Zum Schluss soll noch auf die Modelle E und F verwiesen werden. Diese zeigen auf, dass - wie in vielen anderen Studien - die Veränderungen im Scheidungsverhalten vor allem auf Periodeneffekte und nicht auf Kohorteneffekte zurückzuführen sind. Somit erhält die in diesem Artikel gemachte Annahme, dass bildungsspezifische Unterschiede im Scheidungsverhalten durch ein komplexes Wechselspiel von rechtlichen, institutionellen, kulturellen, ökonomischen und sozialen Kontextfaktoren, welche einen Einfluss auf die Scheidungskosten bzw. den Nutzen einer Ehe für besser gebildete Frauen haben, beeinflusst werden und dass Veränderungen in diesen Kontextfaktoren letztlich zu Veränderungen in den bildungsspezifischen Unterschieden im Scheidungsverhalten führen, Bestätigung.

\section{Diskussion}

Im vorliegenden Artikel wurde der Zusammenhang zwischen dem Bildungsniveau der Frauen und dem Scheidungsverhalten zwischen 1952 und 2013 in der Schweiz mit Daten der Volkszählung 2013 auf Veränderungen hin untersucht. Die unter anderem auf Goodes Theorie der sich verändernden sozialen Determinanten einer Scheidung sowie auf neueren theoretischen Ansätzen zur Transformation des Ehemodells von der Spezialisierung zur Symmetrie basierende Hypothese lautet, dass die Scheidungswahrscheinlichkeit in der Schweiz zwischen 1952 und 2013 für Frauen mit niedrigerer Bildung zu-, für Frauen mit hoher Bildung jedoch (noch) nicht abgenommen hat, sodass sich der Zusammenhang zwischen dem Bildungsniveau der Frauen und dem Scheidungsverhalten im genannten Beobachtungszeitraum zwar abgeschwächt, sich letztlich aber (noch) nicht ins Gegenteil verkehrt hat.

Die Resultate der Discrete-Time-Event-Analysen können wie folgt zusammengefasst werden: Erstens konnten bildungsspezifische Unterschiede im Scheidungsverhalten aufgezeigt werden. Von 1952 bis 1999 war die Scheidungswahrscheinlichkeit für Frauen mit einer höheren Bildung am höchsten. Zweitens hat die Scheidungswahrscheinlichkeit für alle drei Bildungsgruppen zugenommen, jedoch ist sie für Frauen mit einer tiefen Bildung am stärksten angestiegen. Und drittens führt diese Entwicklung dazu, dass ab 2000 keine Unterschiede zwischen den niedrig und den mittel gebildeten Frauen mehr nachweisbar sind und ab 2004 gar keine bildungsspezifischen Unterschiede im Scheidungsverhalten zu vermerken sind. 
Diese Ergebnisse bestätigen die Hypothese. So hat sich die postulierte Zunahme der Scheidungswahrscheinlichkeit der niedriger gebildeten Frauen bewahrheitet, was - wenn auch nicht empirisch getestet - als Hinweis darauf interpretiert werden könnte, dass gesunkene Scheidungskosten zu einer Zunahme der Scheidungswahrscheinlichkeit bei niedriger gebildeten Frauen führen. Ebenfalls bestätigt hat sich, dass die Scheidungswahrscheinlichkeit bei höher gebildeten Frauen (noch) nicht abgenommen hat. Verifiziert hat sich auch die Annahme, dass sich der Zusammenhang zwischen dem Bildungsniveau der Frauen und dem Scheidungsverhalten verändert hat: Er hat sich zwischen 1952 und 2003 abgeschwächt und ist dann ab dem Jahr 2004 ganz verschwunden. Er hat sich jedoch noch nicht - wie in anderen europäischen Ländern - ins Gegenteil verkehrt.

Diese Studie ging davon aus, dass die bildungsspezifischen Unterschiede im Scheidungsverhalten von einem Wechselspiel von rechtlichen, institutionellen, ökonomischen, kulturellen und sozialen Kontextfaktoren beeinflusst werden und eine Veränderung dieser Kontextfaktoren auch zu einer Veränderung in den bildungsspezifischen Unterschieden im Scheidungsverhalten führt. Diese Annahmen erhielten Bestätigung, da die Periodeneffekte stärker als die Kohorteneffekte waren. Die Studie konnte - ausser der Scheidungsrechtsrevision, deren Einfluss auf die bildungsspezifischen Unterschiede im Scheidungsverhalten nicht nachweisbar war die Wirkung der einzelnen Kontextfaktoren nicht empirisch testen, jedoch hat sie einen vertieften Einblick in den sich verändernden Kontext der Schweiz zwischen 1952 und 2013 geliefert und gezeigt, dass sich das Kosten-Nutzen-Modell gut eignet, um diese Veränderungen zu beschreiben und zu interpretieren. Sie hat zudem einen ersten Einblick in die sich über die Zeit verändernden bildungsspezifischen Ungleichheiten im Scheidungsverhalten der Frauen ermöglicht. Die Studie hat jedoch auch Einschränkungen. Da die Daten keine Einkommens- und Erwerbsstatusbiografie enthalten, war es nicht möglich, zwei Faktoren, welche die Kosten und den Nutzen einer Ehe beeinflussen - die Einkommenssituation sowie der Erwerbsstatus der Frauen vor der Scheidung - im Modell zu berücksichtigen.

Es wäre im Anschluss an diese Studie interessant zu sehen, ob sich das KostenNutzen-Modell auch für andere Länder als Analyse- und Interpretationsraster eignet. Insbesondere wäre es aufschlussreich zu untersuchen, ob sich in den egalitäreren europäischen Staaten bereits eine Abnahme der Scheidungswahrscheinlichkeit bei Frauen mit einer hohen Bildung zeigt.

Es wird sich zeigen, ob in der Schweiz die Scheidungswahrscheinlichkeit der Frauen mit einer niedrigen Bildung weiterhin so stark ansteigt und ob jene der Frauen mit einer hohen Bildung aufgrund einer beschleunigten Entwicklung in Richtung egalitäre Geschlechterverhältnisse und symmetrisches Ehemodell tatsächlich abnehmen wird, sodass sich der Zusammenhang zwischen dem Bildungsniveau der Frauen und dem Scheidungsverhalten doch noch ins Gegenteil verkehrt. Eine 
Replikation dieser Studie in ein paar Jahren mit neusten Daten wird eine Antwort auf diese Frage liefern.

Sollte sich die Annahme bewahrheiten, dass sich der Zusammenhang zwischen dem Bildungsniveau der Frauen und dem Scheidungsverhalten in der Schweiz, aber auch in allen anderen europäischen Ländern letztlich ins Gegenteil verkehrt, dann wird dies weitreichende gesellschaftliche Konsequenzen haben. McLanahan (2004) zeigt auf, dass die Elemente des demografischen Wandels, die mit einer Zunahme der Ressourcen (Zeit und Geld) für Kinder verknüpft sind - Alter der Mutter bei der Geburt, Erwerbsbeteiligung der Mutter und Beteiligung des Vaters bei der Kinderbetreuung -, am schnellsten in den Familien der Oberschicht stattfinden, während die Elemente des Wandels, die mit einer Abnahme der Ressourcen verbunden sind alleinerziehender Elternteil und Scheidung -, am schnellsten vonstatten gehen bei Familien mit tieferer Schichtzugehörigkeit. Diese Entwicklungen würden dann zu noch grösseren Unterschieden bezüglich der Ressourcen der Kinder - gemessen an Zeit und Geld der Eltern - führen. Es ist somit wichtig, die bildungsspezifischen Unterschiede im Scheidungsverhalten weiterhin im Auge zu behalten.

\section{$7 \quad$ Literatur}

Ai, Chunrong und Edward C. Norton. 2003. Interaction terms in logit and probit models. Economics Letters 80: 123-129.

Amato, Paul R. 1996. Explaining the intergenerational transmission of divorce. Journal of Marriage and the Family 58: 628-640.

Becker, Gary S. 1981. A Treatise on the Family. Cambridge, MA: Harvard University Press.

Bernardi, Fabrizio und Juan-Ignacio Martinez-Pastor. 2010. Female education and marriage dissolution: is it a selection effect? European Sociological Review 27(6): 693-707.

Bernardi, Fabrizio und Juan-Ignacio Martinez-Pastor. 2011. Divorce risk factors and their variations over time in Spain. Demographic Research 24: 771-800.

BFS (Bundesamt für Statistik). 2005. Fortschritte und Stagnation in der Gleichstellung der Geschlechter 1970-2000. Neuenburg: BFS.

BFS (Bundesamt für Statistik). 2008. Familien in der Schweiz-Statistischer Bericht 2008. Neuenburg: BFS.

BFS (Bundesamt für Statistik). 2009. Demographisches Verhalten der Familien in der Schweiz - 1970 bis 2008. Neuenburg: BFS.

BFS (Bundesamt für Statistik). 2015a. Sprachen, Religionen - Daten, Indikatoren. Neuenburg: BFS, http:// www.bfs.admin.ch/bfs/portal/de/index/themen/01/05/blank/key/religionen.html (13.05.2015).

BFS (Bundesamt für Statistik). 2015b. Vereinbarkeit von Erwerbsarbeit und Familie. Neuenburg: BFS, http://www.bfs.admin.ch/bfs/portal/de/index/regionen/thematische_karten/gleichstellungsatlas/ vereinbarkeit_von_familie_und_erwerbsarbeit/familienmodelle.html (13.05.2015).

BFS (Bundesamt für Statistik). 2015c. Kinderlose Frauen. Neuenburg: BFS, http://www.bfs.admin.ch/ bfs/portal/de/index/regionen/thematische_karten/gleichstellungsatlas/familien_und_haushaltsformen/kinderlosigkeit.html (20.07.2015).

Blossfeld, Hans-Peter, Alessandra de Rose, Jan Hoem und Goetz Rohwer. 1995. Education, modernization, and the risk of marriage disruption in Sweden, West Germany, and Italy. S. 200-222 in 
Gender and Family Change in Industrialized Societies, hrsg. von Karen Oppenheim und An-Magritt Jensen. Oxford: Clarendon Press.

Blossfeld, Hans-Peter und Yossi Shavit. 1993. Persisting barriers: changes in educational opportunities in thirteen countries. S. 1-23 in Persistent Inequality, hrsg. von Yossi Shavit and Hans-Peter Blossfeld. Boulder: Westview Press.

Brambor, Thomas, William Roberts Clark und Matt Golder. 2005. Understanding interaction models: improving empirical analyses. Political Analysis 14: 63-82.

Chan, Tak Win und Brendan Halpin. 2002. Union dissolution in the United Kingdom. International Journal of Sociology 32(4): 76-93.

Charles, Maria. 2005. Entwicklung der beruflichen Segregation nach Geschlecht und nach Staatsangehörigkeit in der Schweiz, 1970-2000. Neuenburg: BFS.

Chen, Wan-Chi. 2012. The changing pattern of educational differentials in divorce in the context of gender egalitarianization: the case of Taiwan. Population Research and Policy Review 31: 831-853.

Cherlin, Andrew J. 1992. Marriage, Divorce, Remarriage. Cambridge, MA: Harvard University Press.

De Graaf, Paul M. und Matthijs Kalmijn. 2006. Change and stability in the social determinants of divorce: a comparison of marriage cohorts in the Netherlands. European Sociological Review 22: 561-572.

Fux, Beat. 2005. Familiale Lebensformen im Wandel. Neuenburg: BFS.

Gonzalez, Libertad und Tarja K. Viitanen. 2009. The effect of divorce laws on divorce rates in Europe. European Economic Review 5: 127-138.

Goode, William J. 1962. Marital satisfaction and instability: a cross-cultural class analysis of divorce rates. S. 377-387 in Class, Status, and Power. Social Stratification in Comparative Perspective, hrsg. von Reinhard Bendix und Seymour M. Lipset. New York: The Free Press.

Goode, William J. 1970. World Revolution and Family Patterns. New York: The Free Press.

Goode, William J. 1993. World Changes in Divorce Patterns. New Haven: Yale University Press.

Haller, Max, Franz Hoellinger und Martin Gomilshak. 2000. Attitudes towards gender roles in international comparison: new findings from twenty countries. S. 131-152 in New Qualities in the Life Course: Intercultural Aspects, hrsg. von Sylvia Supper und Rudolf Richter. Würzburg: Ergon.

Härkönen, Juho. 2005. Divorce risk factors across Finnish marriage cohorts, 1954-1989. Yearbook of Population Research in Finland 41: 151-164.

Härkönen, Juho. 2013. Divorce: trends, patterns, causes, consequences. Working Paper 2013(3). Universität Stockholm, http://www.su.se/polopoly_fs/1.133184.1366922030!/menu/standard/file/ WP_2013_3.pdf (17.12.2015).

Härkönen, Juho und Dronkers Jaap. 2006. Stability and change in the educational gradient of divorce. A comparison of seventeen countries. European Sociological Review 22: 501-517.

Hehli, Simon. 05.05.2015. Kirchenvolk will Segnung homosexueller Paare. Neuste Zürcher Zeitung, http://www.nzz.ch/schweiz/kirchenvolk-will-segnung-schwuler-paare-1.18536363 (15.03.2015).

Hoem, Jan M. 1997. Educational gradients in divorce risks in Sweden in recent decades. Population Studies 51: 19-27.

Hoem, Britta und Jan M. Hoem. 1992. The disruption of marital and non-marital unions in contemporary Sweden. S. 61-93 in Demographic Applications of Event History Analysis, hrsg. von James Trussell, Richard Hankinson und Judith Tilton. England: Claredon Press.

Isen, Adam und Betsey Stevenson. 2010. Women's education and family behavior: trends in marriage, divorce and fertility. NBER Working Paper 15725. National Bureau of Economic Research, http:// www.nber.org/papers/w15725 (13.05.2015).

Lyngstad, Torkild Hovde und Marika Jalovaara. 2010. A review of the antecedents of union dissolution. Demographic Research 23: 257-292.

Martin, Teresa C. und Larry L. Bumpass. 1989. Recent trends in marital disruption. Demography 26: 37-51. 
Masia, Maurizia und Monica Budowski. 2009. Trennung, Scheidung oder Gründung einer neuen Lebensgemeinschaft - Auswirkungen von Erwerbstätigkeit, Bildung und Familienverlauf auf die materielle Lebenssituation. S. 93-112 in Armut trotz Arbeit. Die neue Arbeitswelt als Herausforderung für die Sozialpolitik, hrsg. von Stefan Kutzner, Michael Nollert, Jean M. Bovin und Monica Budowski. Zürich: Seismo.

Matysiak, Anna, Marta Styrc und Daniele Vignoli. 2014. The educational gradient in marital disruption: a meta-analysis of European research findings. Population Studies: A Journal of Demography 68(2): 197-215.

McLanahan, Sara. 2004. Diverging destinies: how children are fading under the second demographic transition. Demography 41: 607-628.

Nicolet, Sarah und Anke Tresch. 2009. Changing religiosity, changing politics? The influence of "belonging" and "believing" on political attitudes in Switzerland. Politics and Religion 2(1): 76-99.

Nordenmark, Mikael. 2013. Disagreement about division of household labour and experiences of work-family conflict in different gender regimes. Multidisciplinary Journal of Gender Studies 2(3): 205-232.

Oppenheimer, Valerie K. 1997. Women's employment and the gain to marriage: the specialization and trading model. Annual Review of Sociology 23: 431-453.

Pfau-Effinger, Birgit. 2005. Wandel der Geschlechterkultur und Geschlechterpolitiken in konservativen Wohlfahrtsstaaten - Deutschland, Österreich und Schweiz. Gender Politik Online, http://www.fu-berlin.de/sites/gpo/tagungen/Kulturelle_Hegemonie_und_Geschlecht_als_ Herausforderung/index.html (20.05.2015).

Poortman, Anne-Rigt und Matthijs Kalmijn. 2002. Women's labour market position and divorce in the Netherlands: evaluating economic interpretations of the work effect. European Journal of Population 18: 175-202.

Salvini, Silvana und Daniele Vignoli. 2011. Things change: women's and men's marital disruption dynamics in Italy during a time of social transformations, 1970-2003. Demographic Research 24(5): 145-174.

Schwartz, Christine R. und Robert D. Mare. 2005. Trends in educational assortative marriage from 1940 to 2003. Demography 42(4): 621-646.

Singer, Judith D. und John B. Willett. 2003. Applied Longitudinal Data Analysis: Modeling Change and Event Occurrence. Oxford: Oxford University Press.

Smith, Ian. 2002. European divorce laws, divorce rates, and their consequences. S. 212-229 in The Law of Economics of Marriage and Divorce, hrsg. von Antony W. Dnes und Robert Rowthorn. Cambridge: Cambridge University Press.

Sobotka, Tomàš und Laurent Toulemon. 2008. Changing family and partnership behaviour: common trends and persistent diversity across Europe. Demographic Research 19(6): 85-138.

South, Scott J. 1995. Do you need to shop around: age at marriage, spousal alternatives, and marital dissolution. Journal of Family Issues 16(4): 432-449.

Stutz, Heidi und Caroline Knupfer. 2012. Absicherung unbezahlter Care-Arbeit von Frauen und Männern. Anpassungsbedarf des Sozialstaats in Zeiten sich ändernder Arbeitsteilung. Bern: Eidgenössisches Büro für die Gleichstellung von Frau und Mann.

Thoenen, Olivia. 2010. Reconciliation of work and family life in Switzerland. German Policy Studies 6(3): 13-48.

Wagner, Michael und Bernd Weiss. 2006. On the variation of divorce risks in Europe: findings from a meta-analysis of European longitudinal studies. European Sociological Review 22: 483-500.

White, Lynn K. 1990. Determinants of divorce: a review of research in the eighties. Journal of Marriage and Family 52(4): 904-912.

Williams, Richard. 2012. Using the margins command to estimate and interpret adjusted predictions and marginal effects. Stata Journal 12(2): 308-331. 